\title{
Influence of a combination of Lactobacillus acidophilus NCFM and lactitol on healthy elderly: intestinal and immune parameters
}

\author{
Arthur C. Ouwehand*, Kirsti Tiihonen, Markku Saarinen, Heli Putaala and Nina Rautonen \\ Health and Nutrition, Danisco, Sokeritehtaantie 20, 02460 Kantvik, Finland \\ (Received 21 June 2007 - Revised 28 April 2008 - Accepted 29 April 2008 - First published online 18 July 2008)
}

With increasing age, a number of physiological changes take place which are reflected in immune and bowel function. These changes may relate to the commonly assumed age-related changes in intestinal microbiota; most noticeably a reduction in bifidobacteria. The current study aimed at modifying the intestinal microbiota with a potential synbiotic on selected immune and microbiota markers. Healthy elderly subjects were randomised to consume during 2 weeks either a placebo (sucrose) or a combination of lactitol and Lactobacillus acidophilus NCFM twice daily in a double-blind parallel trial. After the intervention, stool frequency was higher in the synbiotic group than in the placebo group and a significant increase in faecal $L$. acidophilus NCFM levels was observed in the synbiotic group, after baseline correction. In contrast to the generally held opinion, the study subjects had faecal Bifidobacterium levels that were similar to those reported in healthy young adults. These levels were, nevertheless, significantly increased by the intervention. Levels of SCFA were not changed significantly. Of the measured immune markers, PGE $_{2}$ levels were different between treatments and IgA levels changed over time. These changes were modest which may relate to the fact that the volunteers were healthy. Spermidine levels changed over time which may suggest an improved mucosal integrity and intestinal motility. The results suggest that consumption of lactitol combined with $L$. acidophilus NCFM twice daily may improve some markers of the intestinal microbiota composition and mucosal functions.

Probiotics: Prebiotics: Synbiotics: Lactobacillus acidophilus: Lactitol

With the increase in life expectancy in affluent societies, a growing part of the population in such societies will experience specific age-related disorders. Not all of these will be serious, but may nevertheless affect the quality of life. Common problems encountered in seniors are reduced bowel function and reduced immune function. Both may be associated with changes in the composition and activity of the intestinal microbiota ${ }^{(1,2)}$.

Furthermore, the common use of non-steroidal anti-inflammatory drugs (NSAID) among the elderly may affect their intestinal health. The most common adverse event caused by NSAID is damage to the mucosa in the gastrointestinal tract ${ }^{(3,4)}$. According to a recent study by Hartikainen et al. ${ }^{(5)}$, $70 \%$ of the over 75-year-olds in Kuopio, Eastern Finland (where the current study was performed) were taking at least one analgesic, of which NSAID were the most commonly used. A recent cross-sectional study indicated differences in microbial activity in elderly people, depending on the use of NSAID $^{(6)}$

The common opinion is that seniors have reduced levels of bifidobacteria ${ }^{(7)}$. Whether this really is true or whether this is related to differences in techniques used, culture or molecular-based, or is related to the country where the subjects live ${ }^{(8)}$, remains to be determined. Bifidobacteria are in any case considered to be beneficial members of the intestinal microbiota and maintenance of sufficient high levels of bifidobacteria is considered important in this respect ${ }^{(9)}$.

Specific probiotics and prebiotics are able to increase the level of intestinal bifidobacteria ${ }^{(10,11)}$, and improve bowel function $^{(12)}$ and immune function ${ }^{(13)}$. The current study therefore aimed to investigate the influence of a combination of a probiotic and prebiotic on these parameters in healthy seniors. As probiotic, Lactobacillus acidophilus NCFM was chosen as it has been shown to improve intestinal microbiota metabolism $^{(14)}$ and immune function ${ }^{(15)}$. However, the strain has not been observed to increase faecal bifidobacteria levels ${ }^{(16)}$. As the prebiotic component, lactitol was chosen because it has been shown to be bifidogenic ${ }^{(17)}$ and to increase bowel movements ${ }^{(18)}$. Furthermore, since lactitol has been shown to be a good carbon source for lactobacilli in general and L. acidophilus in particular ${ }^{(19)}$, and is utilised well by L. acidophilus NCFM (results not shown), it could provide a synbiotic combination.

\section{Experimental methods}

Study subjects

The main inclusion criteria of the study subjects were age over 65 years and regular use of NSAID (three or more times

Abbreviations: CFU, colony-forming units; NSAID, non-steroidal anti-inflammatory drugs.

* Corresponding author: Dr Arthur Ouwehand, fax +35892982203, email arthur.ouwehand@danisco.com 
per week). Altogether fifty-one subjects were recruited from the city of Kuopio (Eastern Finland).

The subjects followed their habitual diet during the study. The diet in this part of Finland is typically rich in fibre (for males and females of that age group 25.7 and $21.0 \mathrm{~g} / \mathrm{d}$, respectively) originating mainly from rye bread, whole-grain porridge and berries. To reduce the variation of diets, the study subjects used the meal services offered by a local caterer (Kuopion Ateria). Subjects were asked about their pre-trial use of probiotic and prebiotic products and food products with high fibre content. The intake of fibre-rich food was not found to be different between the two groups. The main source of fibre was rye bread, providing on average $11.6 \mathrm{~g}$ fibre/d for subjects in the test group and $10.7 \mathrm{~g}$ fibre/d for subjects in the placebo group. The use of prebiotic- and probiotic-containing foods was not allowed during the study and was discontinued after the screening visit.

The exclusion criteria were critical illness, inflammatory bowel disease, coeliac disease and a major malignancy in the gastrointestinal tract. The use of antibiotics was not allowed for 1 month prior to and for the whole duration of the study. The inclusion and exclusion criteria were checked during the run-in period before randomisation. Background demographic information on the subjects from both groups is presented in Table 1.

The study was approved by the Research Ethics Committee, Hospital District of Northern Savo (Finland) and written informed consent was obtained from the volunteers.

\section{Study product}

The study product (hereafter referred to as synbiotic) consisted of lactitol (Danisco Sweeteners, Redhill, UK) and Lactobacillus acidophilus NCFM (Danisco Cultures, Madison, WI, USA). Lactitol and L. acidophilus NCFM were milled to the same particle size and mixed to give a concentration of $2 \times 10^{9}$ colony-forming units (CFU)/g. The synbiotic was packed in sachets containing $5 \cdot 0-5 \cdot 5 \mathrm{~g}$. The stability of the product was monitored for 3 months and no significant change in viable numbers was detected (results not shown). The placebo product consisted of $5 \mathrm{~g}$ sucrose milled to the same particle size and packed in identical sachets.

\section{Study design}

Study subjects were randomly assigned to either placebo or synbiotic group. Both study subjects and investigators were blinded to the nature of the product. The study was performed in a parallel manner, with a 2 -week run-in period followed by

Table 1. Subject characteristics

\begin{tabular}{lcc}
\hline & Placebo & Synbiotic \\
\hline No. of subjects & 23 & 24 \\
Age (years) & & \\
$\quad$ Mean & $71 \cdot 7$ & $70 \cdot 3$ \\
SD & $6 \cdot 2$ & $7 \cdot 2$ \\
Sex & 7 & 5 \\
$\quad$ Male & 16 & 19 \\
Female & & \\
\hline
\end{tabular}

a 2-week intervention period and finished with a 2-week wash-out period. Each 2-week periods ended with faecal sampling.

During the intervention period the study subjects were instructed to consume, on two separate occasions during the day, one sachet. The subjects were instructed to mix the test powders with, for example, yogurt or juice. Otherwise the subjects followed their habitual diet.

The subjects were carefully instructed to obtain the faecal samples according to the protocol. All faecal samples were stored in the subjects' home freezer, transferred to the laboratory within $12 \mathrm{~h}$ and stored at $-70^{\circ} \mathrm{C}$ until analysed.

During the study, subjects recorded in a study diary all changes in their medication, health status and bowel function as well as consumption of NSAID in case the consumption pattern was irregular. The use of test products was recorded daily and the records were checked by the study nurse at each visit. The average intake of test products was 1.99 (SD 0.05) sachets/d in the synbiotic group and 1.97 (SD 0.08) sachets/d in the placebo group.

\section{Physico-chemical analyses}

For the analysis of ammonia, $6 \mathrm{ml}$ water were added to $1 \mathrm{~g}$ faeces. After thorough mixing, the sample was centrifuged at $5000 \mathrm{~g}$ for $5 \mathrm{~min}$ and $100 \mu \mathrm{l} 1 \mathrm{M}-\mathrm{NaOH}$ was added to $1 \mathrm{ml}$ of the supernatant. Ammonia was then measured potentiometrically using an $\mathrm{NH}_{3}$ selective electrode (Orion Research). Ammonium chloride was used as calibration standard.

For DM determination, approximately $1 \mathrm{~g}$ faecal sample was weighed, dried at $105^{\circ} \mathrm{C}$ for $16 \mathrm{~h}$, cooled down in a desiccator to room temperature, reweighed and the DM content (\%) was calculated.

Microbial activity was determined through measurement of SCFA and branched-chain fatty acids in faeces. Internal standard (1 $\mathrm{ml} 20 \mathrm{~mm}$-pivalic acid) and $5 \mathrm{ml}$ water were added to $1 \mathrm{~g}$ of the sample. After thorough mixing, the sample was centrifuged at $5000 \mathrm{~g}$ for $5 \mathrm{~min}$. Following centrifugation, $250 \mu \mathrm{l}$ saturated oxalic acid solution was added to $500 \mu \mathrm{l}$ supernatant and the mixture was incubated at $4^{\circ} \mathrm{C}$ for $60 \mathrm{~min}$ and subsequently centrifuged at $16000 \mathrm{~g}$ for $5 \mathrm{~min}$. Supernatant $(1 \mu \mathrm{l})$ was analysed by GC using a glass column packed with 80/120 Carbopack B-DA/4\% on Carbowax $20 \mathrm{M}$ stationary phase (Supelco, Bellefonte, PA, USA) at $175^{\circ} \mathrm{C}$ and with helium as the carrier gas at a flow rate of $24 \mathrm{ml} / \mathrm{min}$. The temperature of the injector and the flame ionisation detector were 200 and $250^{\circ} \mathrm{C}$, respectively. The concentration of acetic acid, propionic acid, butyric acid, isobutyric acid, valeric acid, isovaleric acid, 2-methylbutyric acid and lactic acid was determined.

Biogenic amines in faeces were analysed as described previously by Saarinen ${ }^{(20)}$.

To analyse D- and L-lactic acid, $1 \mathrm{~g}$ sample was weighed in a test tube and $6 \mathrm{ml}$ water were added. The sample was shaken thoroughly and centrifuged at $10000 \mathrm{~g}$ for $10 \mathrm{~min}$. Supernatant $(400 \mu \mathrm{l})$ was transferred into a $1.5 \mathrm{ml}$ microfuge tube. To precipitate protein, $400 \mu \mathrm{l} 0.4 \mathrm{M}-\mathrm{HClO}_{4}$ was added. The sample was kept on ice for $5 \mathrm{~min}$ and centrifuged ( $16000 \mathrm{~g}$ for $5 \mathrm{~min}$ ). To neutralise the sample, $600 \mu \mathrm{l}$ supernatant was transferred into a new $1.5 \mathrm{ml}$ microfuge tube and $70 \mu \mathrm{l} 2 \mathrm{M}-\mathrm{KOH}$ was added. The sample was kept on ice for 
$5 \mathrm{~min}$ and centrifuged $(16000 \mathrm{~g}, 5 \mathrm{~min})$. In the supernatant, D- and L-lactic acid were analysed enzymatically as instructed by the manufacturer (R-Biopharm E1112821).

\section{Microbial analyses}

The total bacteria counts in faecal samples were determined by flow cytometry (FACSCalibur; Becton Dickinson) as described previously ${ }^{(21)}$. In short, bacterial fractions were prepared by suspending faecal samples in sodium phosphate buffer $(50 \mathrm{~mm}, \mathrm{pH} 8)$, followed by centrifugation $(30000 \mathrm{~g}$, $30 \mathrm{~min}$ ); the pellet was washed three more times. The cell samples were diluted, fixed (37\% formaldehyde) and stained with a fluorescent nucleic acid binding dye SYTO 24 (Molecular Probes, Leiden, The Netherlands).

For quantification of Bifidobacterium, L. acidophilus, L. acidophilus NCFM, Clostridium perfringens and sulphatereducing bacteria by real-time PCR (Table 2), DNA was extracted from bacteria isolated from faecal samples using the method described by Apajalahti et al. ${ }^{(22)}$. In short, bacteria were subjected to five freeze-thaw cycles in 2-amino-2-hydroxymethyl-1,3-propanediol (10 mM) EDTA $(1 \mathrm{mM})$ buffer $(\mathrm{pH} 8)$ and subsequently treated with lysozyme and proteinase $\mathrm{K}$. The recovered bacterial DNA was used to enumerate total bifidobacteria with primers and probes designed from 16S rRNA gene as described by Mäkivuokko et al. ${ }^{(23)}$. Primers and probe for the detection of sulphate-reducing bacteria were designed from the adenosine- $5^{\prime}$-phosphosulphate reductase $\alpha$ gene of Desulfovibrio intestinalis using PrimerExpress software (Applied Biosystems, Foster City, CA, USA) as described by Tiihonen et al. ${ }^{(6)}$, and primers and probe detecting $C$. perfringens were designed from phospholipase $\mathrm{C}$ gene as described by Tiihonen et al. ${ }^{(6)}$. Total $L$. acidophilus was determined using primers described by Furet et al. ${ }^{(24)}$ with an in-house designed MGB-probe. L. acidophilus NCFM was quantified using primers and probes designed from the Clustered Regularly Interspaced Short Palindrome Repeats sequence of $L$. acidophilus $\mathrm{NCFM}^{(25)}$ using PrimerExpress software. For the PCR reactions $1 \mu \mathrm{g}$ bacterial DNA was amplified with $300 \mathrm{~nm}$ primers for the analyses for $C$. perfringens and total bifidobacteria, and $900 \mathrm{~nm}$ primers for the analyses for $D$. intestinalis, L. acidophilus NCFM and total L. acidophilus (Medprobe, Normay, Oslo, Norway), and $200 \mathrm{~nm}$ probe (Medprobe or Applied Biosystems) in TaqMan Universal PCR Master Mix (Applied Biosystems). The assays were run on an ABI
PRISM 7000 Sequence Detection System (Applied Biosystems) using the instrument's default settings. To prepare standard curves for absolute quantification, isolated chromosomal DNA either from D. intestinalis (DSM 11275; DSMZ, Braunschweig, Germany), C. perfringens (ATCC 13 124; LGC Promochem AB, Borås, Sweden), Bifidobacterium adolescentis (DSM 20083; DSMZ) or L. acidophilus NCFM (Danisco Cultures) were used. The mass for one bacterial chromosome was calculated by using the Avogadro constant and assuming the mean molecular weight of a base pair to be 650 . Threshold cycle values from standard runs with bacterial chromosomal DNA were plotted against the number of bacteria corresponding to the mass of DNA added to the standard runs at 10 -fold dilutions. The results are expressed as quantity of bacteria/g faeces.

\section{Immunological analyses}

Changes in the immunological status of the intestine were monitored by measuring the concentrations of $\operatorname{IgA}$, TNF- $\alpha$, calprotectin and $\mathrm{PGE}_{2}$ from the soluble fraction of faeces. For IgA, TNF- $\alpha$ and $\mathrm{PGE}_{2}$ measurements, the frozen samples were thawed and extracted with bovine serum albumin as described previously and stored at $-20^{\circ} \mathrm{C}$ before analysis ${ }^{(26)}$. Concentrations of $\operatorname{IgA}, \mathrm{TNF}-\alpha$ and $\mathrm{PGE}_{2}$ were then determined by ELISA according to the manufacturer's instructions (E80-102, Bethyl Laboratories Inc., Montgomery, TX, USA; R\&D Systems Inc., Minneapolis, MN, USA; and Cayman Chemical Company, MI, USA, respectively). The concentrations of calprotectin were determined by ELISA following the manufacturer's instructions (Calpro AS, Oslo, Norway). The results were expressed as $\mu \mathrm{g} / \mathrm{g}$ fresh weight $(\mathrm{IgA}$ and calprotectin) or $\mathrm{pg} / \mathrm{g}$ fresh weight (TNF- $\alpha$ and $\left.\mathrm{PGE}_{2}\right)$.

\section{Statistical analysis}

To determine differences between the groups and over time, data were analysed with repeated measures ANOVA with a linear mixed-effects model, having effects for time, group, and their joint effect. Individual baseline differences were taken into account by including the first time-point as a covariate into the model. Log-transformed data were used when residual plots did not indicate constant variance. L. acidophilus NCFM data could not be analysed as described above and were analysed using baseline corrected data with between-group $t$ tests.

Table 2. Primer and probe sequences as used in the current study

\begin{tabular}{|c|c|c|c|}
\hline Target & Primers $5^{\prime}-3^{\prime}$ & Probe & Reference \\
\hline Lactobacillus acidophilus & $\begin{array}{l}\text { GAT CGC ATG ATC AGC TTA TA } \\
\text { AGT CTC TCA ACT CGG CTA TG }\end{array}$ & CGT AAG CTG TCG CTA TG & 24 \\
\hline Lactobacillus acidophilus NCFM & $\begin{array}{l}\text { TGT GAC CAA AAG CGC TCG TA } \\
\text { GCA AGA GCG GAC ATA GCA AGT T }\end{array}$ & AAA GGG CAG AAA GCT AA & 6 \\
\hline Bifidobacterium & $\begin{array}{l}\text { CCT GGT AGT CCA CGC CGT AA } \\
\text { CAG GCG GGA TGC TTA ACG }\end{array}$ & ATC CAG CAT CCA CCG & 23 \\
\hline Clostridium perfringens & $\begin{array}{l}\text { TTT GGA GAT ATA GAT ACT CCA TAT CAT CCT } \\
\text { GTG CAA AAG TCT CAA ACT TAA CAT GTC }\end{array}$ & TAA TGT TAC TGC CGT TGA T & 6 \\
\hline Sulphate-reducing bacteria & $\begin{array}{l}\text { GGC GCT GAA ATG ACC ATG AT } \\
\text { GGC CGT AAC CGT CCT TGA A }\end{array}$ & TTC GTG CCC GCC CG & 6 \\
\hline
\end{tabular}


Correlations between L. acidophilus NCFM and other parameters were calculated using the Pearson test for both logtransformed and original data. Residuals of the Pearson test were plotted and checked for homogeneity. Data not appearing homogeneous even after $\log$ transformation were analysed with Spearman rank correlation.

To test the dependency of the side-effects to the grouping of the individuals into synbiotic or placebo group, Fisher's exact test was used.

$P$ values smaller than 0.05 were considered significant. All statistical analyses were performed with statistical language $\mathrm{R}$, version 2.6.1 ( $\mathrm{R}$ Foundation for Statistical Computing, Vienna, Austria).

\section{Results}

Forty-seven subjects out of the fifty-one recruited completed the study. Two subjects were prescribed antibiotics before the last sampling and were excluded for that reason. Furthermore one subject, although completing the study protocol otherwise, was excluded from the final tables due to lack of NSAID use during one of the study periods.

At run-in, the groups had a mean defecation frequency of 1.2 (SD 0.9) and 1.3 (SD 0.6) per d for placebo and synbiotic, respectively. At the end of intervention, the mean defecation frequency the placebo group was 1.0 (SD 0.6) per $\mathrm{d}$ while it remained 1.3 (SD 0.5) per $\mathrm{d}$ for the synbiotic group. After correction for the baseline difference, the difference at the end of the intervention was statistically significant $(P=0.009)$.

The results of the intestinal microbiota analyses are shown in Table 3. Differences between the groups and over time were calculated using repeated measures ANOVA with linear mixed-effects model. After correction for baseline differences at the end of the run-in period, there was a difference over time for the total microbial numbers $(P=0.00465)$. The numbers were reduced in both groups towards the wash-out period. Bifidobacteria were higher in the synbiotic group after intervention $\left(7.8 \times 10^{9} \mathrm{CFU} / \mathrm{g}\right)$ as compared to the placebo group $\left(3.8 \times 10^{9} \mathrm{CFU} / \mathrm{g}\right)(P=0.0102$ after baseline correction). Bifidobacterium levels declined for both groups after wash-out to $1.8 \times 10^{9}$ and $1.7 \times 10^{9} \mathrm{CFU} / \mathrm{g}$ for the placebo and synbiotic group, respectively $(P<0.00001$ after baseline correction). The level of sulphate reducers changed significantly over time between the intervention and washout period $(P=0.0058$ after baseline correction); for both groups the mean levels increased (Table 3 ). The differences for $L$. acidophilus NCFM could not be modelled with repeated measures ANOVA with linear mixed-effects differences and were therefore calculated from the log-transformed data using a baseline-corrected pair-wise $t$ test. Mean counts of L. acidophilus NCFM are $1.5 \times 10^{7}$ and $4.8 \times 10^{6} \mathrm{CFU} / \mathrm{g}$ for the placebo and synbiotic group, respectively. However, after log transformation and baseline correction, faecal L. acidophilus NCFM counts at the end of the intervention period were 5.63 and 3.67 , for the synbiotic and placebo group, respectively $(P=0 \cdot 000015)$. No other significant differences or changes in the measured microbiota components were observed.

After baseline correction, no differences in the levels of faecal SCFA and branched-chain fatty acids could be observed

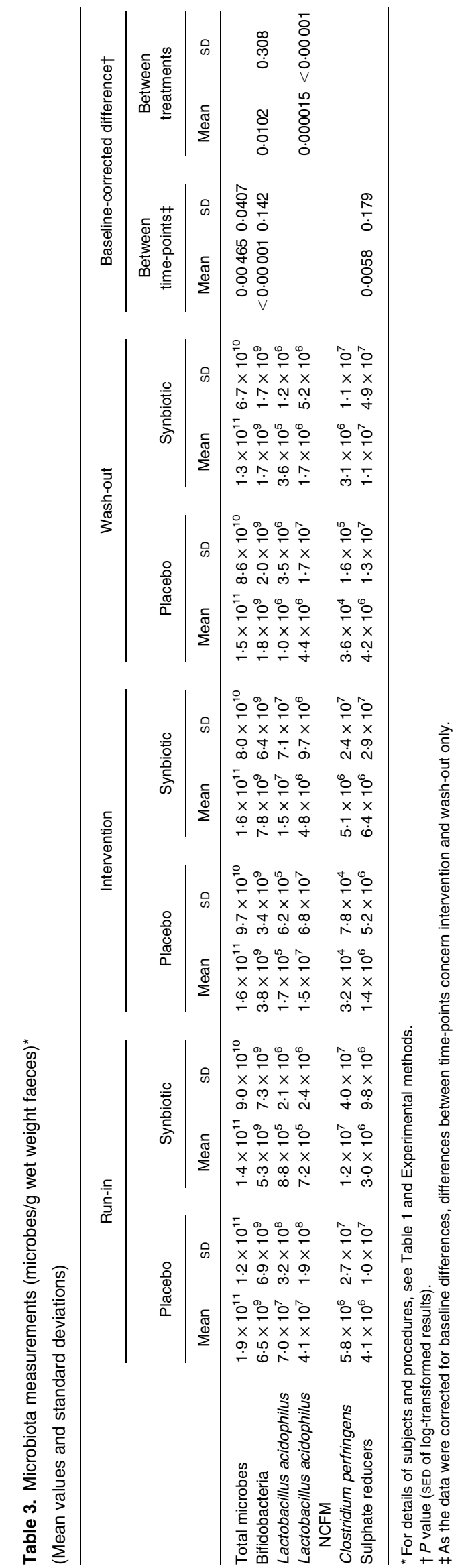


between the two study groups or between intervention and wash-out period (Table 4).

After baseline corrections, there was a change in spermidine levels $(P=0.0325)$, appearing higher in the synbiotic group. In addition, there was a trend for change in faecal $\mathrm{NH}_{3}$ level $(P=0.0914)$. This may relate to the increase in levels in both groups. No other significant differences in levels of DM, ammonia and biogenic amines could be observed between the groups or over time, after correction for baseline difference (Table 5).

Faecal concentrations of IgA were found to change significantly over time after correction for baseline differences $(P=0 \cdot 0241)$. However, there was no difference in IgA concentrations between both groups. In contrast, the concentrations of $\mathrm{PGE}_{2}$ were affected by the synbiotic treatment. Although they tended to be already higher in the synbiotic group during run-in, they were significantly higher even after correcting for this baseline difference $(P=0.0281)$. There was also a trend $(P=0.0821)$ for differences in calprotectin concentrations between the placebo and synbiotic group after correction for baseline differences. No other significant differences in the measured faecal immune markers could be observed between the groups or over time, after correction for baseline difference (Table 6).

Because L. acidophilus NCFM was fed to the volunteers in the synbiotic group, its faecal level was correlated to the other parameters measured at the end of the intervention period. Positive correlations were observed between log-transformed data of L. acidophilus NCFM and histamine (Spearman rank correlation; $R$ 0.419, $P=0.037$ ), and between log-transformed data of $L$. acidophilus NCFM and log-transformed data of spermine (Pearson correlation; $R$ 0.41, $P=0 \cdot 047$ ). During run-in and wash-out, log-transformed data of L. acidophilus NCFM correlated positively with log-transformed data of $L$. acidophilus (Pearson correlation; $R$ 0.73, $P<0.0001$ ).

No significant differences in side-effects, temporary constipation, temporarily increased flatulence, temporary loose stools, combination of diarrhoea, constipation and loose stools, were observed between the two groups during the intervention period.

\section{Discussion}

Elderly subjects have specific ailments. The expected increase in the ageing population in affluent societies may give an increasing burden to health care services. Although many of these cases do not require medication, they may affect their quality of life. The present study investigated the effect of a probiotic and prebiotic combination (synbiotic) of L. acidophilus NCFM and lactitol on parameters of intestinal and immune health, to parameters commonly degenerated at old age. Faecal Bifidobacterium levels, microbial metabolites such as organic acids, branched chain fatty acids and biogenic amines were determined as measures for intestinal microbiota function and bowel function was recorded. Further more, faecal immune markers were determined.

It cannot be avoided that there may be differences at run-in between the study groups for the parameters under investigation. Therefore, a correction of baseline values was applied and all statistical analyses are corrected. Some of the observed changes, from run-in to intervention, may be caused by changes in the environment or indeed be a placebo effect. These are also corrected for by the used analysis model.

One of the main intervention parameters assessed was bowel function. Stool frequency was indeed found to be slightly higher in the synbiotic group as compared to the placebo group. This is in agreement with the observation that lactitol improves bowel function ${ }^{(18)}$. The difference between the groups was modest; this is most likely due to the fact that the recruited subjects had a normal bowel function, with a mean defecation frequency of slightly more than one per day, which was similar to that observed for other NSAID users ${ }^{(6)}$. This most likely relates to the relatively high fibre intake in this population. In this respect, it is also important to note that no side-effects were experienced, although this has been reported for lactitol ${ }^{(27)}$. While not specifically investigated, it was interesting that a number of subjects from the synbiotic group enquired whether and where the product could be purchased as it made them 'feel good'.

Synbiotic intervention increased the level of $L$. acidophilus NCFM numbers, indicating compliance by the volunteers.

Table 4. Faecal SCFA and branched-chain fatty acids $(\mu \mathrm{mol} / \mathrm{g})$ and $\mathrm{D} / \mathrm{L}$-lactic acids $(\mathrm{nmol} / \mathrm{g})^{*}$

(Mean values and standard deviations)

\begin{tabular}{|c|c|c|c|c|c|c|c|c|c|c|c|c|}
\hline & \multicolumn{4}{|c|}{ Run-in } & \multicolumn{4}{|c|}{ Intervention } & \multicolumn{4}{|c|}{ Wash-out } \\
\hline & \multicolumn{2}{|c|}{ Placebo } & \multicolumn{2}{|c|}{ Synbiotic } & \multicolumn{2}{|c|}{ Placebo } & \multicolumn{2}{|c|}{ Synbiotic } & \multicolumn{2}{|c|}{ Placebo } & \multicolumn{2}{|c|}{ Synbiotic } \\
\hline Acetic acid & $40 \cdot 3$ & $13 \cdot 7$ & $44 \cdot 9$ & $15 \cdot 7$ & $42 \cdot 7$ & $15 \cdot 1$ & $41 \cdot 8$ & $13 \cdot 8$ & $40 \cdot 8$ & $15 \cdot 0$ & $47 \cdot 3$ & $17 \cdot 9$ \\
\hline Butyric acid & $11 \cdot 8$ & $7 \cdot 7$ & $14 \cdot 6$ & 8.5 & $12 \cdot 0$ & 5.9 & $12 \cdot 8$ & $6 \cdot 3$ & $11 \cdot 7$ & 6.5 & $15 \cdot 6$ & 8.5 \\
\hline Propionic acid & $11 \cdot 8$ & $7 \cdot 6$ & $12 \cdot 8$ & $5 \cdot 1$ & $12 \cdot 0$ & $5 \cdot 0$ & $12 \cdot 7$ & $5 \cdot 6$ & $11 \cdot 8$ & $5 \cdot 8$ & $12 \cdot 6$ & $5 \cdot 0$ \\
\hline L-Lactic acid & 6.5 & 24 & $71 \cdot 2$ & $239 \cdot 2$ & $335 \cdot 7$ & $899 \cdot 2$ & $231 \cdot 0$ & $517 \cdot 9$ & $552 \cdot 7$ & 351.9 & $562 \cdot 0$ & 358.6 \\
\hline D-Lactic acid & $367 \cdot 1$ & $195 \cdot 5$ & $405 \cdot 8$ & $216 \cdot 0$ & 93.6 & $68 \cdot 7$ & $92 \cdot 8$ & $82 \cdot 8$ & $270 \cdot 8$ & $102 \cdot 1$ & $252 \cdot 0$ & $232 \cdot 6$ \\
\hline Valeric acid & 2.5 & 0.9 & 1.8 & 1.0 & $2 \cdot 2$ & 0.9 & $1 \cdot 7$ & 0.7 & $2 \cdot 1$ & $1 \cdot 1$ & 1.7 & 0.8 \\
\hline Sum of SCFA & $70 \cdot 6$ & $27 \cdot 2$ & $77 \cdot 4$ & $27 \cdot 4$ & $73 \cdot 2$ & $24 \cdot 0$ & $72 \cdot 1$ & $22 \cdot 9$ & $70 \cdot 4$ & $26 \cdot 5$ & $80 \cdot 1$ & $28 \cdot 9$ \\
\hline Iso-valeric acid & $1 \cdot 4$ & 0.5 & $1 \cdot 0$ & 0.5 & $1 \cdot 4$ & 0.7 & $1 \cdot 0$ & 0.5 & 1.4 & 0.8 & $1 \cdot 0$ & 0.5 \\
\hline Iso-butyric acid & $1 \cdot 7$ & 0.6 & 1.4 & 0.6 & $1 \cdot 7$ & $0 \cdot 8$ & $1 \cdot 3$ & $0 \cdot 6$ & $1 \cdot 7$ & 0.9 & $1 \cdot 3$ & 0.6 \\
\hline 2-Methyl butyric acid & $1 \cdot 0$ & 0.4 & 0.7 & 0.4 & $1 \cdot 0$ & 0.6 & 0.7 & 0.4 & $1 \cdot 0$ & 0.6 & 0.7 & 0.4 \\
\hline Sum of branched-chain fatty acids & $6 \cdot 6$ & $2 \cdot 1$ & 4.9 & $2 \cdot 2$ & $6 \cdot 4$ & $2 \cdot 9$ & $4 \cdot 6$ & $2 \cdot 1$ & $6 \cdot 1$ & 3.0 & $4 \cdot 6$ & $2 \cdot 0$ \\
\hline
\end{tabular}

${ }^{*}$ For details of subjects and procedures, see Table 1 and Experimental methods. 
Table 5. Faecal ammonia $(\mu \mathrm{mol} / \mathrm{g})$ and biogenic amines $(\mathrm{nmol} / \mathrm{g})^{\star}$ (Mean values and standard deviations)

\begin{tabular}{|c|c|c|c|c|c|c|c|c|c|c|c|c|c|c|c|c|}
\hline & \multicolumn{4}{|c|}{ Run-in } & \multicolumn{4}{|c|}{ Intervention } & \multicolumn{4}{|c|}{ Wash-out } & \multicolumn{4}{|c|}{ Baseline-corrected difference $\dagger$} \\
\hline & \multicolumn{2}{|c|}{ Placebo } & \multicolumn{2}{|c|}{ Synbiotic } & \multicolumn{2}{|c|}{ Placebo } & \multicolumn{2}{|c|}{ Synbiotic } & \multicolumn{2}{|c|}{ Placebo } & \multicolumn{2}{|c|}{ Synbiotic } & \multicolumn{2}{|c|}{$\begin{array}{c}\text { Between } \\
\text { time-pointsł }\end{array}$} & \multicolumn{2}{|c|}{$\begin{array}{l}\text { Between treat- } \\
\text { ments and } \\
\text { time-points }\end{array}$} \\
\hline & Mean & SD & Mean & SD & Mean & SD & Mean & SD & Mean & SD & Mean & SD & Mean & SD & Mean & SD \\
\hline DM (\%) & $24 \cdot 6$ & 4.9 & $21 \cdot 2$ & 4.5 & $24 \cdot 2$ & 7.3 & 21.6 & 4.4 & $22 \cdot 6$ & 4.4 & 24.4 & $6 \cdot 1$ & & & & \\
\hline $\mathrm{NH}_{3}$ & $18 \cdot 3$ & 9.8 & $15 \cdot 4$ & $8 \cdot 2$ & 18.4 & 11.3 & 16.9 & 9.5 & $22 \cdot 27$ & $12 \cdot 3$ & 17.8 & $7 \cdot 8$ & 0.0914 & 2.85 & & \\
\hline Methylamine & 37.5 & 124.8 & $135 \cdot 2$ & 303.0 & $262 \cdot 6$ & 288.9 & $271 \cdot 2$ & $194 \cdot 3$ & $305 \cdot 9$ & $155 \cdot 1$ & $385 \cdot 3$ & $208 \cdot 4$ & & & & \\
\hline Ethylamine & 0 & 0 & 6.7 & 23.4 & 2.5 & $12 \cdot 1$ & 2.6 & $13 \cdot 1$ & 0 & 0 & 0 & 0 & & & & \\
\hline Tryptamine & 0 & 0 & 0 & 0 & 0 & 0 & 0 & 0 & 0 & 0 & 0 & 0 & & & & \\
\hline Butylamine & 0 & 0 & 0 & 0 & 0 & 0 & 0 & 0 & 0 & 0 & 0 & 0 & & & & \\
\hline Phenylethylamine & 0 & 0 & 0 & 0 & 0 & 0 & 0 & 0 & 0 & 0 & 0 & 0 & & & & \\
\hline 2-Methylbutylamine & $101 \cdot 7$ & 355.4 & 641.5 & 2407.5 & 80.7 & $395 \cdot 1$ & 0 & 0 & 0 & 0 & $18 \cdot 2$ & $69 \cdot 1$ & & & & \\
\hline Putrescine & 655.5 & 2144.8 & 375.7 & $855 \cdot 6$ & 359 & $890 \cdot 4$ & 573.3 & $1756 \cdot 1$ & $580 \cdot 4$ & 485.0 & 544.6 & $1040 \cdot 8$ & & & & \\
\hline Piperidine & $76 \cdot 25$ & 207.9 & $19 \cdot 1$ & 84.3 & 56.8 & 169.1 & $6 \cdot 3$ & $23 \cdot 2$ & $69 \cdot 8$ & 167.9 & $12 \cdot 8$ & 64.0 & & & & \\
\hline Cadaverine & $446 \cdot 3$ & $1731 \cdot 8$ & 257.8 & 655.5 & $260 \cdot 6$ & 814.2 & $328 \cdot 3$ & 950.0 & $427 \cdot 35$ & 206.9 & $265 \cdot 7$ & 499.9 & & & & \\
\hline Histamine & $12 \cdot 2$ & 30.6 & 14.6 & $27 \cdot 7$ & $11 \cdot 1$ & 36.8 & 19.9 & 37.6 & 11.56 & 18.6 & 19.4 & 31.5 & & & & \\
\hline Tyramine & 18.7 & 55.0 & 30.8 & 130.9 & $9 \cdot 1$ & $23 \cdot 2$ & $40 \cdot 3$ & 150.7 & $6 \cdot 1$ & 8.7 & 58.8 & 11.7 & & & & \\
\hline Spermidine & 792.9 & 329.5 & $709 \cdot 2$ & 279.7 & 705 & 333.8 & 825.5 & 368.8 & 684.7 & 353.4 & 674.2 & 11.7 & & & 0.0325 & 0.997 \\
\hline Spermine & $46 \cdot 1$ & 37.2 & 41.3 & $25 \cdot 3$ & 36.1 & 23.7 & 59.5 & 35.2 & 44.8 & 39.6 & $48 \cdot 1$ & 35.7 & & & & \\
\hline
\end{tabular}

${ }^{*}$ For details of subjects and procedures, see Table 1 and Experimental methods.

$\ddagger$ As the data were corrected for baseline differences, differences between time-points concern intervention and wash-out only. 
Curiously, the levels of observed L. acidophilus NCFM were on some occasions higher than observed total L. acidophilus amount. As a possible explanation it can be speculated either that the primers used for detecting total L. acidophilus are not broad enough and/or that primers designed for detecting L. acidophilus NCFM also amplify selected strains other than $L$. acidophilus NCFM. The levels of $L$. acidophilus NCFM strongly correlated with total L. acidophilus, indicating that indeed the same organisms were detected. Synbiotic consumption also increased the faecal level of total bifidobacteria, also this is in agreement with earlier observations for lactitol $^{(17)}$. Bifidobacterium levels were relatively high as compared to some earlier reports ${ }^{(28)}$. This may relate to differences in DNA extraction procedures or indicate differences in microbiota composition in different countries, as has been observed in particular for bifidobacteria ${ }^{(8)}$. The proportion of bifidobacteria from the total microbiota $(0.7-2.8 \%)$ was, however, similar to what has been reported earlier, $1 \cdot 1-3 \cdot 5 \%{ }^{(6,8)}$. Although increases in faecal levels of the consumed probiotic (L. acidophilus NCFM) were observed, the results from the present study do, however, not allow us to conclude whether there was an interaction between both components of the fed synbiotic. Levels of sulphate reducers increased from intervention to wash-out. Since this occurred for both groups, it is not likely to be an effect of the intervention.

As far as the metabolic activity of the intestinal microbiota is concerned, only minor changes in the levels of SCFA and branched-chain fatty acids were observed and these were not different between both treatment groups (Table 4), although the levels were similar as reported earlier in an NSAIDuser population ${ }^{(6)}$. This is in contrast to earlier reported increases in faecal acetic and lactic acid after lactitol supplementation $^{(17,29)}$. A possible explanation for this is that in the current study substantially lower levels of lactitol were consumed than in earlier studies $(2 \times 5$ v. $2 \times 10$ and $3 \times 20$ g, respectively). Of the studied biogenic amines, spermidine levels appeared to be increased at the end of intervention in the synbiotic group. This may therefore be associated with the synbiotic treatment. Furthermore, faecal L. acidophilus NCFM levels correlated positively with spermine. Increase in polyamines such as spermine and spermidine have also been observed to correlate positively with the consumption of Bifidobacterium lactis LMK 512 and has been suggested to be associated with reduced inflammation ${ }^{(30)}$, mutagenicity ${ }^{(31)}$ and improved epithelial cell growth ${ }^{(32)}$.

Although immune senescence has been described in the elderly, the changes in the intestinal immune functions in relation to the intestinal environment have not been studied extensively previously ${ }^{(33)}$. Faecal concentrations of $\operatorname{IgA}$ and TNF- $\alpha$ have been reported not to alter in the elderly population $^{(6,34)}$. Also the calprotectin concentrations appeared normal when compared with those reported in healthy adults ${ }^{(35)}$. Instead $\mathrm{PGE}_{2}$ levels appeared lower in the elderly than in young adults ${ }^{(6)}$. In addition to immune modulation, $\mathrm{PGE}_{2}$ has a central role in the normal physiological gastrointestinal functions including cytoprotection, e.g. against NSAID-induced injury, and motility ${ }^{(36)}$. Reduction in $\mathrm{PGE}_{2}$ concentrations in the elderly may thus indicate lowered motility as well as more vulnerable intestinal mucosa. This is well in line with the reduced motor function in the elderly ${ }^{(37)}$ as 
well as more frequent chronic conditions. It therefore appears to indicate a beneficial change that the consumption of the synbiotic was able to induce - a modest increase in the faecal $\mathrm{PGE}_{2}$ levels, although the concentrations did not quite reach the levels found in young adults during the 2 -week intervention period $^{(6)}$.

In conclusion, the consumption of lactitol in combination with L. acidophilus NCFM twice daily was associated with modest improvement in stool frequency without any side-effects. Furthermore, it increased faecal numbers of L. acidophilus NCFM, bifidobacteria and faecal concentrations of spermidine and $\mathrm{PGE}_{2}$ were increased. The present results indicate improved microbiota composition and mucosal functions.

\section{Acknowledgements}

Jaana Oksanen, Kirsi Stenström, Brita Mäki and Jaana Larsson-Leskelä are acknowledged for skilful technical assistance. Dr Janne Nikkilä is acknowledged for statistical analyses and Dr Essi Sarkkinen, MSc Henna Karvonen and MD Sakari Nieminen for organising the study. Financial support for the study was received from the National Technology Agency of Finland (TEKES). The authors are employees of Danisco. Danisco has partially funded the study and is marketing the components tested: Lactobacillus acidophilus NCFM and lactitol. A. C. O., K. T. and N. R. designed the study, interpreted the results and wrote the manuscript. M. S. performed and oversaw the physico-chemical analyses and wrote the corresponding section of the manuscript. H. P. performed and oversaw the microbial analyses and wrote the corresponding sections of the manuscript.

\section{References}

1. Blaut M, Marteau P \& Miller GD (2006) Probiotics and the intestinal microflora. What impact on the immune system, infections and ageing? Curr Nutr Food Sci 2, 79-95.

2. He T, Harmsen HJM, Raangs GC \& Welling GW (2003) Composition of faecal microbiota of elderly people. Microb Ecol Health Dis 15, 153-159.

3. Bjarnason I, Hayllar J, MacPherson AJ \& Russell AS (1993) Side effects of nonsteroidal anti-inflammatory drugs on the small and large intestine in humans. Gastroenterology 104, 1832-1847.

4. Laine L (2003) Gastrointestinal effects of NSAIDs and coxibs. J Pain Symptom Manage 25, Suppl. 2, S32-S40.

5. Hartikainen S, Mantyselka P, Louhivuori-Laako K, Enlund H \& Sulkava R (2005) Concomitant use of analgesics and psychotropics in home-dwelling elderly people - Kuopio $75+$ study. $\mathrm{Br} \mathrm{J}$ Clin Pharmacol 60, 306-310.

6. Tiihonen K, Tynkkynen S, Ouwehand AC, Ahlroos $\mathrm{T}$ \& Rautonen N (2008) The effect of ageing with and without non-steroidal anti-inflammatory drugs on gastrointestinal microbiology and immunology. Br J Nutr (epublication ahead of print version 18 February 2008).

7. Woodmansey EJ (2007) Intestinal bacteria and ageing. J Appl Microbiol 102, 1178-1186.

8. Mueller S, Saunier K, Hanisch C, et al. (2006) Differences in fecal microbiota in different European study populations in relation to age, gender, and country: a cross-sectional study. Appl Environ Microbiol 72, 1027-1033.
9. Crittenden R (2004) An update on probiotic bifidobacteria. In Lactic Acid Bacteria. Microbiological and Functional Aspects, pp. 125-157 [S Salminen, A von Wright and A Ouwehand, editors]. New York: Marcel Dekker.

10. Ouwehand AC, Nurminen P, Mäkivuokko H \& Rautonen N (2006) Effect of Bifidobacterium lactis 420 on microbiota and immune function. Ital J Food Sci 18, 1-5.

11. Kruse H-P, Kleessen B \& Blaut M (2004) Effect of inulin on faecal bifidobacteria in human subjects. Br J Nutr 82, $375-382$

12. Marteau P, Cuillerier E, Meance S, et al. (2002) Bifidobacterium animalis strain DN-173 010 shortens the colonic transit time in healthy women: a double-blind, randomized, controlled study. Aliment Pharmacol Ther 16, 587-593.

13. Gill H, Rutherfurd KJ \& Cross ML (2001) Dietary probiotic supplementation enhances natural killer cell activity in the elderly: an investigation of age-related immunological changes. J Clin Immunol 21, 264-271.

14. Dunn SR, Simenhoff ML, Ahmed KE, et al. (1998) Effect of oral administration of freeze-dried Lactobacillus acidophilus on small bowel bacterial overgrowth in patients with end stage kidney disease. Reducing uremic toxins and improving nutrition. Int Dairy J 8, 545-553.

15. Foligné B, Nutten S, Grangette C, et al. (2007) Correlation between in vitro and in vivo immune modulatory properties of lactic acid bacteria. World J Gastroenterol 13, 236-243.

16. Varcoe J, Zook C, Sui J, Leighton S, Busta F \& Brady L (2002) Variable response to exogenous Lactobacillus acidophilus $\mathrm{NCFM}^{\circledR}$ consumed in different delivery vehicles. J Appl Microbiol 93, 900-906.

17. Ballongue J, Schumann C \& Quignon P (1997) Effects of lactulose and lactitol on colonic microflora and enzymatic activity. Scand J Gastroenterol 32, Suppl. 222, 41-44.

18. Delas N, Gislon J, Glikmanas M, et al. (1991) Le lactitol dans le traitement de la constipation de l'adulte. Ann Gastroenterol Hepatol (Paris) 27, 231-233.

19. Kontula P, Suihko ML, von Wright A \& Mattila-Sandholm $T$ (1999) The effect of lactose derivatives on intestinal lactic acid bacteria. J Dairy Sci 82, 249-256.

20. Saarinen M (2002) Determination of biogenic amines as dansyl derivatives in intestinal digesta and feces by reversed phase HPLC. Chromatographia 55, 297-300.

21. Apajalahti JHA, Kettunen H, Kettunen A, et al. (2002) Culture-independent microbial community analysis reveals that inulin in the diet primarily affects previously unknown bacteria in the mouse cecum. Appl Environ Microbiol 68, 4986-4995.

22. Apajalahti JHA, Särkilahti LK, Mäki BRE, Heikkinen JP, Nurminen PH \& Holben WE (1998) Effective recovery of bacterial DNA and percent-guanine-plus-cytosine-based analysis of community structure in the gastrointestinal tract of broiler chickens. Appl Environ Microbiol 64, 4084-4088.

23. Mäkivuokko H, Nurmi J, Nurminen P, Stowell J \& Rautonen N (2005) In vitro effects on polydextrose by colonic bacteria and caco-2 cell cyclooxygenase gene expression. Nutr Cancer 52, 94-104.

24. Furet J-P, Quénée P \& Taolliez P (2004) Molecular quantification of lactic acid bacteria in fermented milk products using real-time PCR. Int J Food Microbiol 97, 197-207.

25. Altermann E, Russell WM, Azcarate-Peril MA, et al. (2005) Complete genome sequence of the probiotic lactic acid bacterium Lactobacillus acidophilus NCFM. Proc Natl Acad Sci U S A 102, 3906-3912.

26. Peuranen S, Tiihonen K, Apajalahti J, Kettunen A, Saarinen M $\&$ Rautonen N (2004) Combination of polydextrose and lactitol affects microbial ecosystem and immune responses in rat gastrointestinal tract. Br J Nutr 91, 905-914. 
27. Morgan MY, Alonso M \& Stanger LC (1989) Lactitol and lactulose for the treatment of subclinical hepatic encephalopathy in cirrhotic patients. A randomised, cross-over study. J Hepatol $\mathbf{8}$, $208-217$

28. Hopkins MJ \& Macfarlane GT (2002) Changes in predominant bacterial populations in human faeces with age and with Clostridium difficile infection. $J$ Med Microbiol 51, 448-454.

29. Hotten P, Marotta F, Naito Y, et al. (2003) Effects of probiotics, lactitol and rifaximin on intestinal flora and fecal excretion of organic acids in cirrhotic patients. Chin J Digest Dis $\mathbf{4}$, $13-18$.

30. Matsumoto M \& Benno Y (2006) Anti-inflammatory metabolite production in the gut from the consumption of probiotic yogurt containing Bifidobacterium animalis subsp. lactis LKM512. Biosci Biotechnol Biochem 70, 1287-1292.

31. Matsumoto M \& Benno Y (2004) Consumption of Bifidobacterium lactis LKM512 yogurt reduces gut mutagenicity by increasing gut polyamine contents in healthy adult subjects. Mutat Res 568, 147-153.
32. Osborne DL \& Seidel ER (1989) Microflora-derived polyamines modulate obstruction-induced colonic mucosal hypertrophy. Am J Physiol 256, G1049-G1057.

33. Guigoz Y, Dore J \& Schiffrin EJ (2008) The inflammatory status of old age can be nurtured from the intestinal environment. Curr Opin Clin Nutr Metab Care 11, 13-20.

34. Arranz E, O'Mahony S, Barton JR \& Ferguson A (1992) Immunosenescence and mucosal immunity: significant effects of old age on secretory IgA concentrations and intraepithelial lymphocyte counts. Gut 33, 882-886.

35. Maiden L, Thjodleifsson B, Theodors A, Gonzalez J \& Bjarnason I (2005) A quantitative analysis of NSAID-induced small bowel pathology by capsule enteroscopy. Gastroenterology 128, 1172-1178.

36. Dey I, Lejeune M \& Chadee K (2006) Prostaglandin E2 receptor distribution and function in the gastrointestinal tract. Br J Pharmacol 149, 611-623.

37. Madsen JL \& Graff J (2004) Effects of ageing on gastrointestinal motor function. Age Ageing 33, 154-159. 